\title{
A New Family of Somos-like Recurrences
}

\author{
Paul Heideman \\ University of Wisconsin \\ Madison, WI 53705 \\ ppheideman@wisc.edu
}

\author{
Emilie Hogan \\ Department of Mathematics \\ Rutgers, The State University of New Jersey \\ Piscataway, NJ 08854-8019 \\ eahogan@math.rutgers.edu
}

Submitted: Sep 26, 2007; Accepted: Apr 1, 2008; Published: Apr 10, 2008

Mathematics Subject Classification: 11B37

\begin{abstract}
We exhibit a three parameter infinite family of quadratic recurrence relations inspired by the well known Somos sequences. For one infinite subfamily we prove that the recurrence generates an infinite sequence of integers by showing that the same sequence is generated by a linear recurrence (with suitable initial conditions). We also give conjectured relations among the three parameters so that the quadratic recurrences generate sequences of integers.
\end{abstract}

\section{Introduction}

It is always the case that quadratic recurrence relations generate sequences of rational numbers, given that the first finitely many terms are 1 . That there exist quadratic recurrences which generate only integers is very surprising. Michael Somos, through investigations of elliptic theta functions, discovered one such quadratic recurrence which began a new wave of research into this phenomenon [4]. After Somos' discovery [6] that the recurrence

$$
S(n)=\left(S(n-1) S(n-5)+S(n-2) S(n-4)+S(n-3)^{2}\right) / S(n-6)
$$

generated integers when given initial conditions $S(i)=1$ for $0 \leq i \leq 5$, a surge of research began on related sequences. Most importantly for the goals of our problem was the sequence discovered by Dana Scott defined by the recurrence

$$
D(n)=(D(n-1) D(n-3)+D(n-2)) / D(n-4)
$$

with initial conditions $D(i)=1$ for $0 \leq i \leq 3$. This recurrence differs from Somos' original recurrence as it mixes quadratic and linear terms. However, it also defines a sequence consisting only of integers. 
The above two sequences possess an even stronger property than integrality. If the initial conditions are given as $S(m)=x_{m}\left(\right.$ resp. $\left.D(m)=x_{m}\right)$ for $0 \leq m \leq 5$ (resp. $0 \leq$ $m \leq 3)$, these recurrences produce Laurent polynomials. The set of Laurent polynomials are defined in [7] as

$$
\mathbb{R}\left[t, t^{-1}\right]=\left\{\sum a_{i} t^{i} \mid i \in \mathbb{Z}, a_{i} \neq 0 \text { for finitely many } i \in \mathbb{Z}\right\}
$$

We will define Laurent polynomials in more than one variable in the same manner as above. That is, the set of Laurent polynomials in the variables $\left\{x_{i}\right\}_{i=1}^{n}$ is defined to be

$$
\begin{aligned}
& \mathbb{R}\left[\left\{x_{i}\right\}_{i=1}^{n},\left\{x_{i}^{-1}\right\}_{i=1}^{n}\right]= \\
& \quad\left\{\sum a_{i}\left(\prod_{j=1}^{n} x_{j}^{n_{j}}\right) \mid i, n_{j} \in \mathbb{Z}, a_{i} \neq 0 \text { for finitely many } i \in \mathbb{Z}\right\}
\end{aligned}
$$

This Laurent property is discussed in Fomin and Zelevinsky's article [3], and has been used to extract combinatorial information from sequences.

The work done by Dana Scott was important to our discovery since it gave us the idea to probe for sequences defined by a mixture of quadratic and linear terms, rather than just the Somos-like quadratic recurrences. Additionally, work done by Reid Barton [1] on a similar sequence resulted in a combinatorial interpretation, which led us to believe that a similar interpretation of our sequences exists.

\section{Statement of Problem}

While looking at the family of quadratic recurrences given by

$$
a(n) a(n-k)=a(n-1) a(n-k+1)+a(n-(k-1) / 2)+a(n-(k+1) / 2)
$$

with initial conditions $a(m)=1$ for $m \leq k$ we noticed that they seemed to produce a sequence of integers for any odd value of $k$ we chose. By substituting $k=2 K+1$ we can transform the recurrence so it is valid for any positive integer value of $K$. This defines the following recurrence relation

$$
a(n) a(n-(2 K+1))=a(n-1) a(n-2 K)+a(n-K)+a(n-K-1)
$$

now with initial conditions $a(m)=1$ for $m \leq 2 K+1$.

For instance, when $K=1$ the recurrence becomes

$$
a(n) a(n-3)=a(n-1) a(n-2)+a(n-1)+a(n-2)
$$

generating the sequence $\{a(n)\}=\{1,1,1,3,7,31,85 \ldots\}$.

We have proven the integrality assertion by showing that the sequence produced by the quadratic recurrence also satisfies a linear recurrence with integer coefficients and integer initial conditions. It has also been shown that when the initial conditions are given as formal variables the recurrences produce Laurent polynomials (see [5]). Our eventual goal is to find a combinatorial interpretation (e.g. counting perfect matchings of certain graphs). 


\section{Proof of Integrality}

In order to prove that the quadratic recurrence produces integers we show that the sequence also satisfies a linear recurrence with integer coefficients.

Theorem 1. The recurrence

$$
a(n) a(n-(2 K+1))=a(n-1) a(n-2 K)+a(n-K)+a(n-K-1)
$$

with initial conditions $a(m)=1$ for $m \leq 2 K+1$ generates an infinite sequence of integers.

Lemma 1. The initial $6 K+1$ terms of the sequence given by the quadratic recurrence relation (4) satisfy the following conditions:

$$
\begin{array}{rlrl}
a(i)= & 1 & 1 \leq i \leq 2 K+1 \\
a(2 K+i)= & -1+2 i & 2 \leq i \leq K+1 \\
a(3 K+i)= & 1+2 K-2 i+2 i^{2} & 2 \leq i \leq K+1 \\
a(4 K+i)= & -3-8 K+2 i-2 K^{2}+12 K i+2 i^{2}+4 K^{2} i & 2 \leq i \leq K+1 \\
a(5 K+i)= & 3+10 K-10 i+16 K^{2}-16 K i+8 i^{2} & \\
& +4 K^{3}-4 K^{2} i+16 K i^{2}+4 K^{2} i^{2} & & 2 \leq i \leq K+1
\end{array}
$$

Proof. Notice that $a(i)=1$ for $1 \leq i \leq 2 K+1$ by definition. Each of the other relations is proved independently by induction. All initial relations were originally conjectured and proved via a computer program written by Doron Zeilberger [8]. We will now show the proof for $a(2 K+i)=-1+2 i$ with $2 \leq i \leq K+1$.

Base case $i=2$ : We need to verify that $a(2 K+2)=3$. From the quadratic definition of the sequence we have

$$
\begin{aligned}
a(2 K+2) a(1) & =a(2 K+1) a(2)+a(K+2)+a(K+1) \\
a(2 K+2) \cdot 1 & =1 \cdot 1+1+1 \\
\Rightarrow a(2 K+2) & =3
\end{aligned}
$$

Now we assume, as the inductive hypothesis, that $a(2 K+i)=-1+2 i$. We need to show that $a(2 K+(i+1))=-1+2(i+1)=2 i+1$ for $2 \leq i \leq K$. We know that

$$
a(2 K+(i+1)) a(i)=a(2 K+i) a(i+1)+a(K+(i+1))+a(K+i)
$$

Since $2 \leq i \leq K$, we have

$$
\begin{array}{rlrl}
3 & \leq i+1 & & \leq K+1 \\
K+3 & \leq K+(i+1) & \leq 2 K+1 \\
K+2 & \leq & K+i & \leq 2 K
\end{array}
$$

Therefore $a(i+1)=a(K+(i+1))=a(K+i)=1$.

$$
\begin{aligned}
& a(2 K+(i+1)) \cdot 1=(-1+2 i) \cdot 1+1+1 \\
& \Rightarrow a(2 K+(i+1))=2 i+1
\end{aligned}
$$


So by induction, $a(2 K+i)=-1+2 i$ for $2 \leq i \leq K+1$.

Proofs of the other initial relations follow the same series of steps, and will therefore be left up to the reader to verify.

Lemma 2. If the sequence $\{a(n)\}$ is given by the quadratic recurrence (4), then it also satisfies the linear recurrence given by

$$
a(n)=\left[2 K^{2}+8 K+4\right](a(n-2 K)-a(n-4 K))+a(n-6 K)
$$

for all $n \geq 6 K+2$, where the initial $6 K+1$ values are taken to be the first $6 K+1$ values of the quadratic recurrence (4).

Proof. First note that by uniqueness, proving the converse (i.e.- that the sequence given by the linear recurrence (5) satisfies the quadratic recurrence (4)) is equivalent to proving that the statement itself. Thus we prove that the linear recurrence given by (5) satisfies the quadratic recurrence given by (4) using strong induction. Define the sequence $\{a(n)\}$ recursively for all $n \geq 6 K+2$ by (5) and let $a(n)$ for $1 \leq n \leq 6 K+1$ be defined by the initial conditions given in Lemma 1 . For simplicity in notation, let the term $2 K^{2}+8 K+4$ be called $A(K)$. The proof will use methods analogous to those used by Hal Canary [2] to prove integrality of the Dana Scott recurrence (1).

Let

$$
\phi(n)=a(n) a(n-(2 K+1))-a(n-1) a(n-2 K)-a(n-K)-a(n-K-1)
$$

We wish to prove by induction that $\phi(n)=0$ for all $n \in \mathbb{Z}^{+}$. Clearly for $1 \leq n \leq 6 K+1$, $\phi(n)=0$ since the first $6 K+1$ terms are defined to be the first terms given by $(4)$.

For the base case we must prove that $\phi(6 K+2)=0$. This is nothing but algebraic calculations easily verified by a computer algebra system such as Maple or Mathematica.

$$
\phi(6 K+2)=a(6 K+2) a(4 K+1)-a(6 K+1) a(4 K+2)-a(5 K+2)-a(5 K+1)
$$

where we can substitute the initial conditions for the linear recurrence for all but $a(6 K+2)$.

$$
\begin{aligned}
& a(4 K+1)=a(3 K+(K+1))=2 K^{2}+4 K+1 \\
& a(6 K+1)=a(5 K+(K+1))=4 K^{4}+24 K^{3}+40 K^{2}+16 K+1 \\
& a(4 K+2)=6 K^{2}+16 K+9 \\
& a(5 K+2)=4 K^{3}+24 K^{2}+42 K+15 \\
& a(5 K+1)=a(4 K+(K+1))=4 K^{3}+6 K^{2}+10 K+1
\end{aligned}
$$

And $a(6 K+2)$ must be given by the linear recurrence $(5)$

$$
\begin{aligned}
a(6 K+2) & =\left(2 K^{2}+8 K+4\right)(a(4 K+2)-a(2 K+2))+a(2) \\
& =\left(2 K^{2}+8 K+4\right)\left(\left(6 K^{2}+16 K+9\right)-3\right)+1 \\
& =12 K^{4}+80 K^{3}+164 K^{2}+112 K+25
\end{aligned}
$$


Now it is a matter of plugging this into your favorite computer algebra system and verifying that $\phi(6 K+2)=0$.

Since the base case is verified we can proceed with the induction. We make the strong induction assumption that $\phi(m)=0$ for $m<n$. We need to show that $\phi(n)=0$ given the inductive hypothesis. Now, compute $\phi(n)$ by substituting for $a(n), a(n-1), a(n-K)$, and $a(n-K-1)$ from the definition of $\{a(n)\}$.

$$
\begin{aligned}
a(n) & =A(K) a(n-2 K)-A(K) a(n-4 K)+a(n-6 K) \\
a(n-1) & =A(K) a(n-2 K-1)-A(K) a(n-4 K-1)+a(n-6 K-1) \\
a(n-K) & =A(K) a(n-3 K)-A(K) a(n-5 K)+a(n-7 K) \\
a(n-K-1) & =A(K) a(n-3 K-1)-A(K) a(n-5 K-1)+a(n-7 K-1)
\end{aligned}
$$

After substituting into $\phi(n)$, expand and then simplify and we are left with

$$
\begin{aligned}
\phi(n)= & -A(K) a(n-4 K) a(n-2 K-1)+A(K) a(n-4 K-1) a(n-2 K) \\
& -A(K) a(n-3 K)-A(K) a(n-3 K-1)+a(n-2 K-1) a(n-6 K) \\
& -a(n-2 K) a(n-6 K-1)+A(K) a(n-5 K)+A(K) a(n-5 K-1) \\
& -a(n-7 K)-a(n-7 K-1)
\end{aligned}
$$

You can see that

$$
\begin{aligned}
& -A(K) a(n-4 K) a(n-2 K-1)+A(K) a(n-4 K-1) a(n-2 K)+ \\
& \quad-A(K) a(n-3 K)-A(K) a(n-3 K-1)=-A(K) \phi(n-2 K)
\end{aligned}
$$

which equals 0 by the induction hypothesis. Thus

$$
\begin{aligned}
\phi(n)= & a(n-2 K-1) a(n-6 K)-a(n-2 K) a(n-6 K-1)+ \\
& +A(K) a(n-5 K)+A(K) a(n-5 K-1)-a(n-7 K)+ \\
& -a(n-7 K-1)
\end{aligned}
$$

Substitute for $a(n-2 K)$ and $a(n-2 K-1)$ from the definition of $\{a(n)\}$.

$$
\begin{aligned}
a(n-2 K)= & A(K) a(n-4 K)-A(K) a(n-6 K)+a(n-8 K) \\
a(n-2 K-1)= & A(K) a(n-4 K-1)-A(K) a(n-6 K-1)+ \\
& +a(n-8 K-1)
\end{aligned}
$$

Simplify again to obtain

$$
\begin{aligned}
\phi(n)= & A(K) a(n-4 K-1) a(n-6 K)-A(K) a(n-4 K) a(n-6 K-1)+ \\
& -A(K) a(n-5 K)-A(K) a(n-5 K-1)+ \\
& +a(n-6 K) a(n-8 K-1)-a(n-6 K-1) a(n-8 K)+ \\
& -a(n-7 K)-a(n-7 K-1) \\
= & -A(K) \phi(n-4 K)+\phi(n-6 K) \\
= & 0
\end{aligned}
$$

Thus by induction $\phi(n)=0$ for all $n \in \mathbb{Z}^{+}$. 
Theorem 1 now follows directly from the two lemmas. The first $6 K+1$ terms of the sequence generated by the quadratic recurrence must be integers since they are given by polynomials with integer coefficients in the variables $K$ and $i$. The rest of the terms in the sequence satisfy a linear recurrence with integer coefficients and integer initial conditions. Thus the terms must all be integers.

\section{Laurentness}

Now, instead of declaring that the initial conditions of a recurrence, $r(n)$, be all 1 , we will set $r(i)$ to be the variable $x_{i}$ for the first sufficiently many terms. If all terms in a sequence are Laurent polynomials in the initial variables $\left\{x_{i}\right\}$ then we say that the sequence (or the recurrence producing the sequence) has the Laurent property. In Section 1, we gave examples of some sequences which were conjectured to be integers, and that have been shown to possess the Laurent property. Notice that a sequence having the Laurent property immediately proves integrality of that sequence. Since definition (2) is equivalent to defining the set of Laurent polynomials to be the subset of rational functions in which the denominator is a monomial, setting all variables equal to 1 in a Laurent polynomial clearly produces an integer. For this reason, and because the Laurent property has been helpful in producing a combinatorial proof of integrality in some cases, many people prefer proofs of Laurentness versus other proofs of integrality. In [3], Fomin and Zelevinsky give easily verifiable sufficient conditions for a recurrence to possess the Laurent property. Using these conditions one can prove that the sequence given by (4) with initial conditions $a(i)=x_{i}$ for $1 \leq i \leq 2 K+1$ has the Laurent property. For the proof see [5]. We do not include the proof here because the machinery used, namely Fomin and Zelevinsky's conditions via cluster algebras, are more advanced than our proof via linear recurrence. The technique of showing that a sequence generated by a quadratic recurrence also satisfies a linear recurrence, as in the proof from Section 3, has the potential to be applied in instances where the recurrence in question does not satisfy Fomin and Zelevinsky's conditions but still possesses the Laurent property, or even when the recurrence satisfies integrality without Laurentness.

\section{Generalization of this Family of Quadratic Recur- rences}

There is evidence to suggest that an even broader family of quadratic recurrence relations produces integer sequences. Let the sequence $\{b(n)\}_{n=1}^{\infty}$ be generated by the following recurrence.

$$
b(n) b(n-k)=b(n-i) b(n-k+i)+b(n-j)+b(n-k+j)
$$

with the conditions that $i<k-i<k, j<k-j<k$, and $b(l)=1$ for all $l<k$. With certain constraints on $k, i$, and $j$, the sequence $\{b(n)\}_{n=1}^{\infty}$ is conjectured to be an integer sequence. 
Conjecture 1. Consider the general form of the quadratic recurrence (7) with initial terms $b(l)=1$ for $0 \leq l \leq k$.

- In the case where $k$ is even:

- If $i$ is odd, then $j=\frac{k}{2}$ defines a recurrence that generates only integers.

- If $i$ is even, then $j=\frac{i}{2}, j=\frac{k}{2}$, and $j=\frac{k-i}{2}$ define recurrences that generate only integers.

- In the case where $k$ is odd:

- If $i$ is odd then $j=\frac{k-i}{2}$ defines a recurrence that generates only integers.

- If $i$ is even then $j=\frac{i}{2}$ defines a recurrence that generates only integers.

Furthermore, all other values of $j$ do not define a recurrence that gives integers exclusively.

For example, the recurrence (4) is a special case of the generalization (7) where $k$ is odd, $i=1$, and $j=\frac{k-1}{2}$ in agreement with Conjecture 1 .

\section{Lifting the recurrences to 2-dimensional space}

As mentioned in Section 4, these recurrences satisfy even stronger conditions than integrality. However, the Laurent polynomials which are produced will have increasingly large coefficients. For the purposes of extracting combinatorics, it is preferable to modify these recurrences so that the sequences are Laurent polynomials with all coefficients of 1 (which we will call faithful polynomials) so that each term counts some object (e.g. a perfect matching).

Conjecture 2. For all conjectured families of recurrences in section 4 except for the case where $k$ is even and $j=\frac{k}{2}$, the two dimensional recurrence

$$
\begin{aligned}
T(n, k) T(n-k, k)= & T(n-i, k+2) T(n-k+i, k-2)+ \\
& +T(n-j, k+1)+T(n-k+j, k-1)
\end{aligned}
$$

with initial terms $T(i, j)=x_{i, j}$ for all $i<0$ will generate faithful Laurent polynomials in $x_{i, j}$.

Note that when $k$ is even and $j=\frac{k}{2}$, we still obtain a Laurent polynomial with this method, but by combining linear terms one can see immediately that it is not faithful.

Though it is not entirely clear what this lifting process will give us, it has been a means for finding combinatorics in similar sequences such as the Reid Barton and Dana Scott recurrences [1]. 


\section{Conclusion}

The integrality proof given here only sheds light on a small fraction of this new family of quadratic recurrences. It is our hope to eventually prove that all of the sequences generated by the quadratic recurrences in Conjecture 1 satisfy linear recurrences, and thus are integer sequences. The fact that the Laurent property is also satisfied in this special case (and possibly in the other cases of Conjecture 1) may lead to the discovery of some family of combinatorial objects which are counted by this family of integer sequences.

\section{Acknowledgements}

We would like to thank James Propp for suggesting possible paths to take in this research and for running the Spatial Systems Laboratory at the University of Wisconsin-Madison where all of our research was conducted. Thanks also goes to the rest of the Spatial Systems Laboratory members for their helpful contributions along the way. We would also like to acknowledge and extend thanks to the NSF's Research Experiences for Undergraduates program and the NSA for funding our research. Finally we would like to thank Doron Zeilberger of Rutgers University for helping us make the final leap in the formulation and proof of Lemma 1.

\section{References}

[1] Reid Barton. Personal communication, 2004.

[2] Hal Canary. Personal communication, 2004.

[3] Sergey Fomin and Andrew Zelevinsky. The Laurent phenomenon. Adv. in Appl. Math, 28(2):119-144, 2002.

[4] D. Gale. Mathematical entertainments: The strange and surprising saga of the Somos sequences. Math. Intel., 13:40-42, 1991.

[5] Emilie Hogan. An infinite family of quadratic recurrences satisfying the Laurent phenomenon. In preparation, 2008.

[6] Michael Somos. Somos 6-sequence. http://grail.csuohio.edu/ somos/somos6.html.

[7] Eric Weisstein. Laurent polynomial, MathWorld-A Wolfram Web Resource. http://www.mathworld.com/LaurentPolynomial.html.

[8] Doron Zeilberger. Maple program "EmiliePaul(k,n)".

http://www.math.rutgers.edu/ zeilberg/tokhniot/EMILIE. 